\title{
Constructing a Proton Titration Curve from lon-Step Measurements, Applied to a Membrane with Adsorbed Protein
}

\author{
Jan C. T. Eijkel, Coen Bosch, Wouter Olthuis, ${ }^{1}$ And Piet Bergveld \\ MESA Research Institute, University of Twente, Box 217, 7500AE, Enschede, The Netherlands
}

Received July 5, 1996; accepted November 11, 1996

\begin{abstract}
A new measuring method is described for obtaining a proton titration curve. The curve is obtained from a microporous composite membrane, consisting of polystyrene beads in an agarose matrix, with lysozyme molecules adsorbed to the bead surface. The membrane is incorporated into a sensor system by deposition on a silicon chip with a $\mathrm{pH}$-sensitive ion-sensitive field effect transistor (ISFE T) located in the middle of a Ag/A gC I electrode. The actual measurement is performed by creating a stepwise change in the salt concentration of the bathing electrolyte (the ion step) and measuring the ISFE T potential versus the $\mathrm{Ag} / \mathrm{AgCl}$ electrode. This potential shows a transient change in the ion step, which indicates a transient $\mathrm{pH}$ change in the membrane. This procedure is repeated at a series of $\mathrm{pH}$ values. Equations are presented to calculate the proton titration curve of the membrane from the amplitude and duration of the measured transients. Measurements show qualitative agreement between the curves obtained and equilibrium titration experiments on the same system. 1997 Academic Press

Key Words: proton titration; ion-sensitive field effect transistor; polystyrene bead; lysozyme; concentration step; $\mathrm{Ag} / \mathrm{AgCl}$ electrode.
\end{abstract}

\section{INTRODUCTION}

Recently, an ion-sensitive field effective transistor (ISFET)-based immunosensor was developed $(1,2)$. The sensor device has also been used to determine protein and heparin concentrations (3-5). It consists of a $\mathrm{pH}$-sensitive ISFET in the middle of a pCl-sensitive $\mathrm{Ag} / \mathrm{AgCl}$ electrode (6). On top of both ion sensors is deposited a microporous composite membrane consisting of polystyrene beads in an agarose matrix (see Fig. 1). The device is placed in a flow-through system and the potential of the ISFET versus the $\mathrm{Ag} / \mathrm{AgCl}$ electrode is recorded. A measurement is performed by increasing in a stepwise manner the $\mathrm{KNO}_{3}$ concentration in the electrolyte (the ion step), while keeping the $\mathrm{pH}$ and $\mathrm{pCl}$ constant. On the ion step the ISFET potential shows a transient change. This transient is modulated by the adsorption of charged macromolecules to the device membrane.

\footnotetext{
${ }^{1}$ To whom correspondence should be addressed.
}

Previously it was shown that the potential transient is determined by a proton release or uptake in the membrane (7). In this paper the theoretical relationships are derived between the amplitude and the duration of the transients and the proton titration curve of the membrane at a certain $\mathrm{pH}$. Using these relationships, and measuring the potential transients at a series of $\mathrm{pH}$ values, it will be possible to construct a titration curve and thus to obtain information on adsorbed macromolecules. For biosensor purposes, the relationships can also be applied in the inverse direction; i.e., the device response can be predicted from known titrational behavior of the different components. In this paper the adsorption of the positively charged protein lysozyme is studied as a model.

The measurements presented can be regarded as an extension of common practice in protein titration, namely, the relative positioning of the titration curves at different ionic strengths by using the $\mathrm{pH}$ change that results from a change in ionic strength $(8,9)$. In this type of experiment, the $\mathrm{pH}$ change is measured at only one initial $\mathrm{pH}$ value. In the investigations presented the method is extended by measuring at a series of $\mathrm{pH}$ values. The extension is made possible by the transient and reversible nature of the ion-step measurements.

\section{THEORY RELATING ION-STEP MEASUREMENT RESULTS TO PROTON TITRATION BEHAVIOR}

\subsection{General Assumptions}

The membranes under consideration consist of polystyrene beads of 112-nm diameter in an agarose matrix. The membrane has small pores (estimated dimensions from 10 to $100 \mathrm{~nm}$ ) and a large internal polystyrene surface. For the description of the proton titration of this surface and of the processes occurring on an ion step, the following assumptions are made:

1. The specific adsorption of ions other than protons is negligible.

2. The agarose has a net zero charge in the $\mathrm{pH}$ range investigated, and shows no proton association. The assump- 


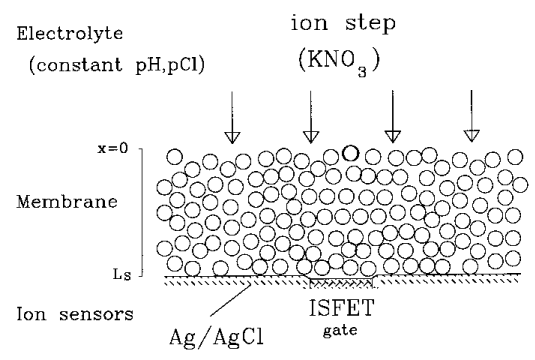

FIG. 1. Cross-sectional representation of the measuring device.

tion is justified since the type of agarose used is characterized by a zero endosmotic flow in the $\mathrm{pH}$ range investigated.

3. The bead surface can be represented by an isolated flat polystyrene surface, since the Debye length is negligible in relation to the bead diameter at the salt concentrations used (10).

4. A smeared-out charge model can be used for the surface charge.

5. The electrical double layer does not extend to the pore center or the ion sensor surfaces.

6. The system can be described as one dimensional in the direction perpendicular to the sensor surface. This is justified since the pore diameter is small enough to ensure a constant equilibrium in the plane parallel to the sensor surface. The electrolyte/membrane interface is located at $x=0$, the ion sensor surfaces at $x=L_{\mathrm{S}}$ ( see Fig. 1).

7. The rise in membrane $\mathrm{KNO}_{3}$ concentration on an ion step is considered immediate. This is acceptable since the rise in the $\mathrm{KNO}_{3}$ concentration at the electrolyte/membrane interface and the subsequent $\mathrm{KNO}_{3}$ diffusion through the membrane are completed in about $1 \mathrm{~s}$, which is negligible on the scale of the measured transient (3).

8. The amount of protons in the double layers can be neglected with respect to the amount of protons in the electroneutral pore solution.

9. Both ion sensors react Nernstian and instantaneous to the concentration of their potential-determining ion at $x=$ $L_{\mathrm{S}}$.

\subsection{Local and Temporal Proton Association Equilibrium}

Based on assumptions 5 and 6 , the relationship between local and temporal $\mathrm{pH}$ in the pore center and the protondissociated fraction $\alpha$ of wall-fixed groups $j$ can be represented by the equilibrium equation (11)

$$
\begin{aligned}
\mathrm{pH}(t, x)= & \mathrm{p} K_{\mathrm{a}, \text { int }, j} \\
& +\mathrm{p} K_{a, \text { med }, j}+\mathrm{p} K_{\mathrm{a}, \mathrm{el}, j}+\log \frac{\alpha_{j}(t, x)}{1-\alpha_{j}(t, x)},
\end{aligned}
$$

where $t$ is time (s), $x$ is the coordinate perpendicular to the sensor surface $(\mathrm{m})$, the superscript e refers to the electrolyte bulk, and the subscripts int, med, and el respectively refer to the intrinsic, medium, and electrical contributions to $\mathrm{p} K_{a}$. The medium term contains all effects exerted by the microenvironment of polystyrene and agarose.

The dissociation of a proton will result in the formation of a negatively charged group when starting from a neutral one or a neutral group when starting from a protonated base. Summation of the charge contributions of all dissociated and nondissociated groups of all types $j$ gives the wall charge density $\sigma^{\mathrm{w}}\left(\mathrm{C} \mathrm{m}^{-2}\right)$,

$$
\begin{aligned}
\sigma^{\mathrm{w}}(t, x)=F \sum_{j} c_{\mathrm{TOT}, j}^{\mathrm{w}}\left\{z_{j}(1-\right. & \left.\alpha_{j}(t, x)\right) \\
& \left.+\left(z_{j}-1\right) \alpha_{j}(t, x)\right\},
\end{aligned}
$$

where the superscript $\mathrm{w}$ refers to the pore wall, $z_{j}$ is the charge of a nondissociated ion of group $j, c_{\mathrm{TOT}, j}^{\mathrm{w}}$ is the total wall concentration of groups of type $j\left(\mathrm{~mol} \mathrm{~m}^{-2}\right)$, and $F$ is the Faraday constant $\left[\mathrm{C} \mathrm{mol}^{-1}\right]$.

The electrical term in Eq. [1] is proportional to the potential difference between the wall surface and the electrolyte bulk, $\Phi^{\mathrm{w}}(t, x)-\Phi^{\mathrm{e}}$

$$
\mathrm{p} K_{a, \mathrm{el}, j}=\frac{-F\left(\Phi^{\mathrm{w}}(t, x)-\Phi^{\mathrm{e}}\right)}{2.3 R T},
$$

where $\Phi$ is the electric potential [V], and $R$ and $T$ have their usual meaning. By substituting in Eq. [1], we obtain the following for the proton dissociation equilibrium of groups of type $j$ :

$$
\begin{aligned}
\mathrm{pH}(t, x)=\mathrm{p} K_{a, \mathrm{int}, j}-\frac{F\left(\Phi^{\mathrm{w}}(t, x)-\Phi^{\mathrm{e}}\right)}{2.3 R T} & \\
& +\log \frac{\alpha_{j}(t, x)}{1-\alpha_{j}(t, x)} .
\end{aligned}
$$

$\Phi^{\mathrm{w}}-\Phi^{\mathrm{e}}$ can be calculated from the wall charge density $\sigma^{\mathrm{w}}$. When a diffuse-layer model is used for the electrical double layer, their relationship in the presence of a 1:1 salt is $(12,13)$

$$
\left(\Phi^{\mathrm{w}}(t, x)-\Phi^{\mathrm{e}}\right)=\frac{2 R T}{F} \cdot \sinh ^{-1}\left(\frac{\sigma^{\mathrm{w}}(t, x)}{\sqrt{8 R T \epsilon_{0} \epsilon_{\mathrm{r}} c^{\mathrm{e}}}}\right),
$$

where $c^{\mathrm{e}}$ is the salt concentration in the electrolyte (mol $\left.\mathrm{m}^{-3}\right), \epsilon_{0}$ is the permittivity of free space $\left(\mathrm{C} \mathrm{V}^{-1} \mathrm{~m}^{-1}\right)$, and $\epsilon_{\mathrm{r}}$ is the relative permittivity of water. Proton titration curves describe $\sigma^{\mathrm{w}}$ as a function of $c^{\mathrm{e}}$ and $\mathrm{pH}^{\mathrm{e}}$, and are obtained by solving Eqs. [2], [4], and [5]. The treatment given here is identical to the classic treatment for the titration of dissolved proteins (14-16) except for Eq. [5], which in the 


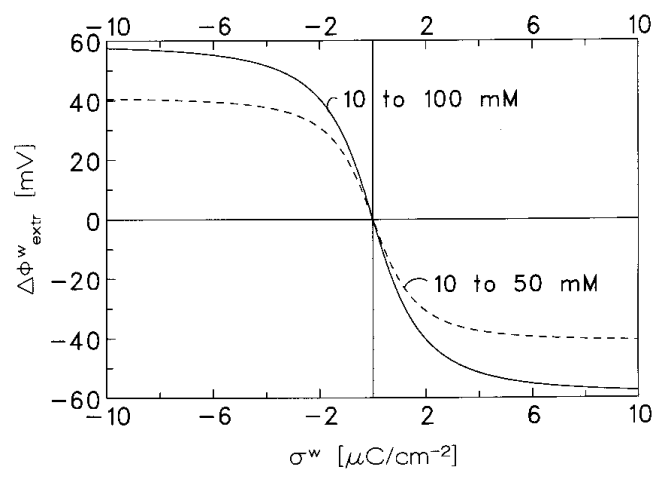

FIG. 2. Extreme change in the wall potential as a function of the wall charge density for ion steps from 10 to 50 and from 10 to $100 \mathrm{mM}$.

classic treatment is replaced by the Debye-Hückel approximation (17).

Apparent quantities. The electrical contribution to $\mathrm{p} K_{a}$ as formulated in Eq. [3] can be included in the $\mathrm{p} K_{a}$ of the associating groups, resulting in the definition of an apparent $\mathrm{p} K_{a}(10)$ :

$$
\mathrm{p} K_{a, \text { app }, j}=\mathrm{p} K_{a, \mathrm{int}, j}-\frac{F\left(\Phi^{\mathrm{w}}-\Phi^{\mathrm{e}}\right)}{2.3 R T} .
$$

The apparent $\mathrm{p} K_{a}$ decreases with decreasing salt concentration. Analogously, an apparent wall buffer capacity $\beta_{\text {app }}^{\mathrm{w}}$ $\left(\mathrm{mol} \mathrm{m}{ }^{-2}\right)$ is defined, proportional to the slope of the titration curve and decreasing with decreasing salt concentration:

$$
\beta_{\mathrm{app}}^{\mathrm{w}}=-\frac{\partial \sigma^{\mathrm{w}}}{F \partial \mathrm{pH}^{\mathrm{e}}}
$$

\subsection{R elation between the Titration C urve and the Ion-Step Transients}

At the stepwise rise in pore $\mathrm{KNO}_{3}$ concentration, the apparent $\mathrm{p} K_{a}$ values of the membrane groups change due to the change in the wall potential (see Eq. [6]). The consequent immediate reequilibration across the pores and the subsequent reequilibration between membrane and electrolyte will involve local and temporal changes of $\mathrm{pH}, \mathrm{pCl}$, and dissociated fraction of groups. The changes in $\mathrm{pH}$ and $\mathrm{pCl}$ and their influence on the measured potential transient are considered in this subsection.

\subsubsection{The Measuring Ion Sensors}

The measuring signal is the output voltage of the ISFET amplifier while grounding the electrolyte with the $\mathrm{Ag} / \mathrm{AgCl}$ electrode. A change in the measured potential reflects a change in the ISFET gate-oxide $\left(\mathrm{Ta}_{2} \mathrm{O}_{5}\right)$ potential versus the $\mathrm{Ag} / \mathrm{AgCl}$ potential. This potential can be considered to stem from the electrochemical cell $\mathrm{Cu}^{\prime} / \mathrm{Si} / \mathrm{Ta}_{2} \mathrm{O}_{5} / \mathrm{H}^{+}, \mathrm{Cl}^{-} /$
$\mathrm{AgCl} / \mathrm{Ag} / \mathrm{Cu}^{\prime \prime}(18)$. The potential transient on an ion step, $\Delta \Phi^{\mathrm{s}}(t)$, is defined relative to the potential at $t<0$, and is determined by the temporal change in the $\mathrm{H}^{+}$and $\mathrm{Cl}^{-}$ concentrations at the surface of the ion sensors. The transient is given by

$$
\Delta \Phi^{\mathrm{s}}(t)=\frac{R T}{F} \ln \frac{c_{\mathrm{H}}\left(t, L_{\mathrm{s}}\right) \cdot c_{\mathrm{Cl}}\left(t, L_{\mathrm{s}}\right)}{c_{\mathrm{H}}^{\mathrm{e}} \cdot c_{\mathrm{Cl}}^{\mathrm{e}}},
$$

considering that the concentration values at $t<0$ equal the values in the bulk electrolyte, $c_{\mathrm{H}}^{\mathrm{e}}$ and $c_{\mathrm{Cl}}^{\mathrm{e}}$ (assumption 5). As will theoretically be shown in Subsection 2.3.3, the change in chloride concentration on the ion step can be neglected (6). We then obtain for the potential transient

$$
\begin{aligned}
\Delta \Phi^{\mathrm{s}}(t) & =\frac{R T}{F} \ln \frac{c_{\mathrm{H}}\left(t, L_{\mathrm{s}}\right)}{c_{\mathrm{H}}^{\mathrm{e}}} \\
& =-\frac{2.3 R T}{F} \cdot \Delta \mathrm{pH}\left(t, L_{\mathrm{s}}\right) .
\end{aligned}
$$

Thus, the measured transient depends on the temporal change in $\mathrm{pH}$ at the ISFET surface. An equation for the extreme $\mathrm{pH}$ change and hence the extreme of the potential transient is derived in Subsection 2.3.2.a. The time constant for the decay phase is derived in Subsection 2.3.2.b.

\subsubsection{Pore $p H$ during the Ion Step}

a. Extreme change in the wall potential and the pore $\mathrm{pH}$. Assumption 6 states a constant equilibrium across the pores. Thus, the change in the wall potential on an ion step will be followed by an immediate change in the proton concentration at the pore walls and hence in the dissociation equilibrium of the wall-fixed groups. Protons are released from, or taken up by, wall-fixed groups. The change in wall potential will reach an extreme when the resulting change in dissociated fractions $\alpha_{j}$ of all buffering groups is negligible. This extreme change can be deduced from Eq. [5] as

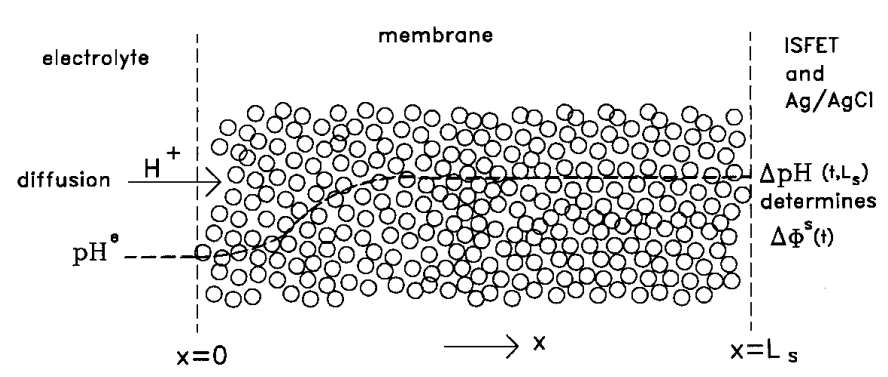

FIG . 3. Positively charged membrane during proton reequilibration with the electrolyte. For $\mathrm{K}^{+}, \mathrm{NO}_{3}^{-}$, and $\mathrm{Cl}^{-}$equilibrium is already attained, but the proton reequilibration proceeds much more slowly due to the buffering action of the wall-fixed groups. In the final situation the $\mathrm{pH}$ in the electroneutral pores returns to $\mathrm{pH}^{\mathrm{e}}$. 


$$
\Delta \Phi_{\mathrm{extr}}^{\mathrm{w}}=\frac{2 R T}{F} \cdot \ln \left(\sqrt{\frac{c^{\mathrm{e}^{\prime \prime}}}{c^{\mathrm{e}^{\prime}}}} \cdot \frac{s^{\mathrm{w}}+\sqrt{\left(s^{\mathrm{w}}\right)^{2}+c^{\mathrm{e}^{\prime}}}}{s^{\mathrm{w}}+\sqrt{\left(s^{\mathrm{w}}\right)^{2}+c^{\mathrm{e}^{\prime \prime}}}}\right),
$$

where $s^{\mathrm{w}}=\sigma^{\mathrm{w}} / \sqrt{8 R T \epsilon_{0} \epsilon_{\mathrm{r}}}=269 \sigma^{\mathrm{w}}\left(\mathrm{mol}^{0.5} \mathrm{~m}^{-1.5}\right)$, and $c^{\mathrm{e}^{\prime}}$ and $c^{\mathrm{e}^{\prime \prime}}$ are the salt concentrations at $t=0$ and $t=\infty$. This relationship is represented in Fig. 2 for ion steps of different magnitude. The sign of the wall potential change is the opposite of the sign of the wall charge density. At high $\sigma^{\mathrm{w}}$ the wall potential change approaches a value of $R T / F \cdot \ln \left(c^{\mathrm{e}^{\prime \prime}} / c^{\mathrm{e}^{\prime}}\right)$.

The extreme change in pore $\mathrm{pH}$ that occurs due to proton uptake or release can be calculated from Eq. [4] for constant $\alpha_{j}$ and is proportional to the extreme change in the wall potential:

$$
\Delta \mathrm{pH}_{\mathrm{extr}}=-\frac{F}{2.3 R T} \Delta \Phi_{\mathrm{extr}}^{\mathrm{w}} .
$$

The change in the dissociated fraction $\alpha_{j}$ will be negligible if the buffering of the wall $\mathrm{pH}$ by the wall-fixed groups is much more effective than the buffering of the pore $\mathrm{pH}$ by the local protons. This condition is expressed by

$$
\beta_{\text {app }}^{\mathrm{w}} \gg \beta^{\mathrm{p}} \cdot \frac{(1-p) r}{3 p},
$$

where $\beta^{\mathrm{p}}$ is the buffer capacity of the membrane pore solution $\left(\mathrm{mol} \mathrm{m}^{-3}\right)$. The second term on the right-hand side of Eq. [12] expresses the amount of pore volume per wall area in terms of $p$ (the polystyrene bead volume fraction in the membrane) and $r$ [the bead radius $(\mathrm{m})]$.

b. Pore $\mathrm{pH}$ in the reequilibration phase. The change in pore $\mathrm{pH}$ due to the immediate equilibration across the pores is followed by a slower proton reequilibration between membrane and bulk electrolyte. An arbitrary moment during this phase is shown in Fig. 3 for a positively charged membrane. In this membrane the decrease in pore $\mathrm{pH}$ due to proton influx is delayed by the association of the arriving protons with the wall groups (causing an increase in $\sigma^{\mathrm{w}}$ ). To this diffusion/reaction process the following modified form of Fick's second law of diffusion can be applied [cf. (19, p. 326)]:

$$
\frac{\partial c_{\mathrm{H}}(t, x)}{\partial t}=\frac{D_{\mathrm{H}}}{K(t, x)+1} \cdot \frac{\partial^{2} c_{\mathrm{H}}(t, x)}{\partial x^{2}} .
$$

Here $D_{\mathrm{H}}$ is the diffusion coefficient of the pore protons $\left(\mathrm{m}^{2}\right.$ $\mathrm{s}^{-1}$ ), and

$$
\begin{aligned}
K(t, x) & =\frac{3 p}{(1-p) r} \cdot \frac{\partial \sigma^{\mathrm{w}}(t, x)}{\partial c_{\mathrm{H}}(t, x)} \\
& =\frac{3 p}{(1-p) r} \cdot \frac{\beta_{\mathrm{app}}^{\mathrm{w}}(t, x)}{2.3 c_{\mathrm{H}}(t, x)}
\end{aligned}
$$

The term $K(t, x)$ is a scaling factor for the proton membrane diffusion coefficient, representing the retardation of proton diffusion due to association with fixed groups. Equation [14] shows that $K$ is proportional to the ratio of the buffer capacities of the wall-fixed groups and pore solution. $K$ will on an ion step be a function of both time and place. The contribution of the hydroxyl ions to the ionic fluxes is neglected, which means that Eq. [13] can be applied only at $\mathrm{pH}^{\mathrm{e}}<7$.

To solve Eq. [13], a constant $K$ is assumed. Its formulation is given at the end of this subsection. Protons diffuse from $x=0$ to $x=L_{\mathrm{S}}$. The boundary condition at $x=0$ is

$$
c_{\mathrm{H}}(t, 0)=c_{\mathrm{H}}^{\mathrm{e}} \quad(t \geqslant 0) .
$$

The membrane proton concentration at $t=0$ follows from Eq. [12], assuming that an extreme change in the wall potential is reached:

$$
c_{\mathrm{H}}(0, x)=c_{\mathrm{H}}^{\mathrm{e}} \cdot \exp \left(\frac{F \Delta \Phi_{\mathrm{extr}}^{\mathrm{w}}}{R T}\right) \quad\left(0 \leqslant x \leqslant L_{\mathrm{S}}\right) .
$$

The solution to Eq. [13] for $c_{\mathrm{H}}\left(t, L_{\mathrm{S}}\right.$ ) (at the location of the ISFET), under the conditions of Eqs. [15] and [16], is given by (19)

$$
\begin{aligned}
c_{\mathrm{H}}\left(t, L_{\mathrm{S}}\right)= & c_{\mathrm{H}}^{\mathrm{e}} \cdot\left\{1+\left[\exp \left(\frac{F \Delta \Phi_{\mathrm{extr}}^{\mathrm{w}}}{R T}\right)-1\right]\right. \\
& \left.\times \frac{4}{\pi} \sum_{n=0}^{\infty} \frac{(-1)^{n}}{2 n+1} \exp \left[-\frac{(2 n+1)^{2} t}{\tau}\right]\right\},
\end{aligned}
$$

where

$$
\tau=\frac{4(K+1) \cdot L_{\mathrm{S}}^{2}}{D_{\mathrm{H}} \pi^{2}}(\mathrm{~s})
$$

The value of $K$ is derived by substituting in Eq. [14] an average apparent buffer capacity and an average pore proton concentration. The buffer capacity is averaged over the $\mathrm{pH}$ trajectory traversed. The average pore proton concentration change is derived from Eq. [16] by taking for the average wall potential change half the value of its extreme. Thus,

$$
K=\frac{3 p}{r(1-p)} \cdot \frac{\bar{\beta}_{\mathrm{app}}^{\mathrm{w}}}{2.3 c_{\mathrm{H}}^{\mathrm{e}} \cdot \exp \left(F \Delta \Phi_{\mathrm{extr}}^{\mathrm{w}} / 2 R T\right)} .
$$

\subsubsection{Pore $\mathrm{pCl}$ during the Ion Step}

The immediate ionic reequilibration across the pores on the ion step will also cause a change in pore $\mathrm{pCl}$, which according to Eq. [8] will influence the device transient; however, theoretically the time constant $\tau$ for chloride diffusion 


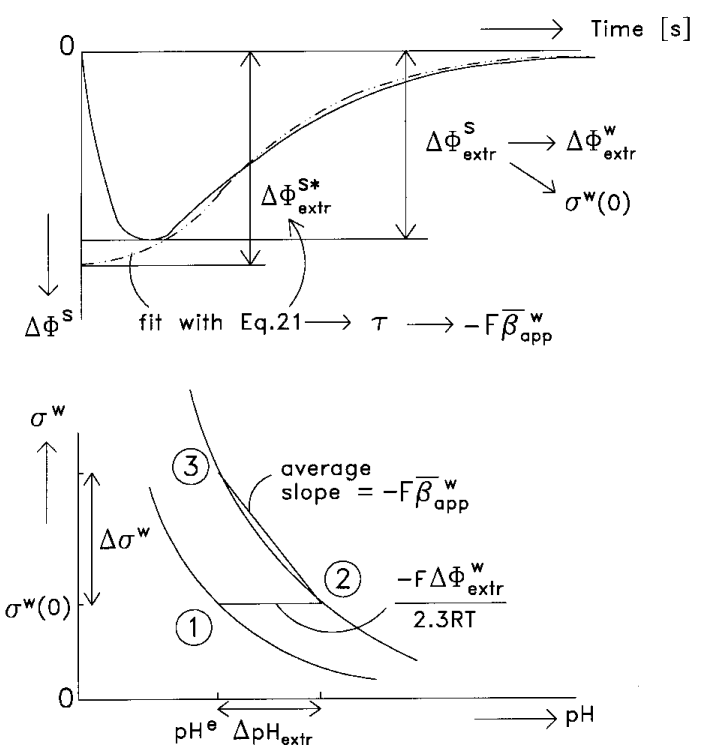

FIG. 4. Relationships between the variables measured by the ion step and titration curve variables.

is small enough to assume that chloride concentration remains constant. Calculated using Eq. [18] with $K=0$ (no $\mathrm{Cl}^{-}$association) and $\mathrm{Cl}^{-}$diffusion coefficient in the pores 0.4 times the value in the electrolyte, $\tau$ is about $32 \mathrm{~ms}$ in the $8-\mu \mathrm{m}$ membranes and $128 \mathrm{~ms}$ in the $16-\mu \mathrm{m}$ membranes used.

\subsubsection{Construction of the Titration Curves}

Figure 4 shows the relationships between a measured transient and the membrane titration curves at low and high salt concentration. The numbers in the bottom part refer to (1) the equilibrium situation at the low salt concentration, (2) the situation directly after the equilibration across the pores, and (3) the new equilibrium situation after completion of the reequilibration with the electrolyte. On an ion step the system moves from (1) to (3).

a. Distance between the curves at $c^{e^{\prime}}$ and $c^{e^{\prime \prime}}$ along the pH axis. According to Eq. [9], the extreme of the device potential transient will be proportional to the extreme of the pore $\mathrm{pH}$ change. This $\mathrm{pH}$ change, in turn, is related to the extreme change in wall potential via Eq. [12]:

$$
\Delta \Phi_{\mathrm{extr}}^{\mathrm{s}}=-\frac{2.3 R T}{F} \cdot \Delta \mathrm{pH}_{\mathrm{extr}}=\Delta \Phi_{\mathrm{extr}}^{\mathrm{w}}
$$

This equality reflects the mediating action of the mobile protons, which can communicate the wall potential change to the membrane pores and finally to the ISFET surface because they are buffered so well by the groups at the pore walls. Since $\Delta \Phi_{\text {extr }}^{\mathrm{w}}$ is defined as the wall potential change at constant $\alpha$ for all groups $j, \Delta \mathrm{pH}_{\text {extr }}$ represents the distance between the two membrane titration curves at constant (initial) fixed charge density, as shown in Fig. 4. In Eq. [20] the actually measured value $\Delta \Phi_{\text {extr }}^{\mathrm{s}}$ can be used or the value $\Delta \Phi_{\text {extr }}^{\text {s* }}$ can be obtained from a curve fit with Eq. [21], which is derived below.

$b$. Wall charge density at $c^{e^{\prime}}$. The wall charge density at $t=0, \sigma^{\mathrm{w}}(0)$, can also be calculated from $\Delta \Phi_{\mathrm{extr}}^{\mathrm{w}}$, by applying Eq. [10] (see Fig. 4). As indicated by the slope of the curve in Fig. 2, the accuracy of this determination will become less at higher potential extremes.

c. Average wall buffer capacity at $c^{e^{\prime \prime}}$. Substituting Eq. [17], which describes the temporal proton concentration at $x=L_{\mathrm{S}}$, into Eq. [8] for constant $c_{\mathrm{Cl}}$, and using the equality expressed in Eq. [20], we obtain an equation that describes the potential transient in time:

$$
\begin{aligned}
\Delta \Phi^{\mathrm{s}}(t)= & \frac{R T}{F} \ln \left\{1+\left[\exp \left(\frac{F \Delta \Phi_{\mathrm{extr}}^{\mathrm{s} *}}{R T}\right)-1\right]\right. \\
& \left.\times \frac{4}{\pi} \sum_{n=0}^{\infty} \frac{(-1)^{n}}{2 n+1} \exp \left[-\frac{(2 n+1)^{2} t}{\tau}\right]\right\} .
\end{aligned}
$$

Equation [21] is suitable for curve fitting on the measured transient curve with fitting parameters $\Delta \Phi_{\text {extr }}^{\mathrm{s} *}$ and $\tau$. From the value obtained for $\tau$, the average apparent wall buffer capacity can be calculated using the following equation, which is derived from Eqs. [18], [19], and [20]:

$$
\begin{aligned}
\bar{\beta}_{\mathrm{app}}^{\mathrm{w}}=\tau \cdot \frac{\pi^{2} D_{\mathrm{H}^{+}}^{\mathrm{P}}}{4 L_{\mathrm{S}}^{2}} \cdot\left[\frac{2.3 r(1-p)}{3 p} \cdot c_{\mathrm{H}^{+}}^{\mathrm{e}}\right. \\
\left.\quad \times \exp \left(\frac{F \Delta \Phi_{\mathrm{extr}}^{\mathrm{s} *}}{2 R T}\right)+1\right] .
\end{aligned}
$$

d. Distance between the curves at $c^{e^{\prime}}$ and $c^{e^{\prime \prime}}$ along the $\sigma^{w}$ axis. Finally, the increase in $\sigma^{\mathrm{w}}$ from (2) to (3) in Fig. 4 can be derived by multiplying the mean slope of the titration curve between (2) and (3), which represents the average apparent wall buffer capacity at $c^{\mathrm{e}^{\prime \prime}}$, with the extreme change in pore $\mathrm{pH}$, and relating the latter to the extreme potential transient by Eqs. [13] and [23]:

$$
\Delta \sigma^{\mathrm{w}}=\Delta \mathrm{pH}_{\mathrm{extr}} \cdot-F \bar{\beta}_{\mathrm{app}}^{\mathrm{w}}=\frac{F^{2} \Delta \Phi_{\mathrm{extr}}^{\mathrm{s}}}{2.3 R T} \cdot \bar{\beta}_{\mathrm{app}}^{\mathrm{w}} .
$$

\subsubsection{Correcting for the Influence of the ISFET Gate- Oxide Response}

In another paper it was shown that a bare ISFET (without a membrane) shows a potential transient on an ion step, with an amplitude of $10-30 \mathrm{mV}$ and a duration of 


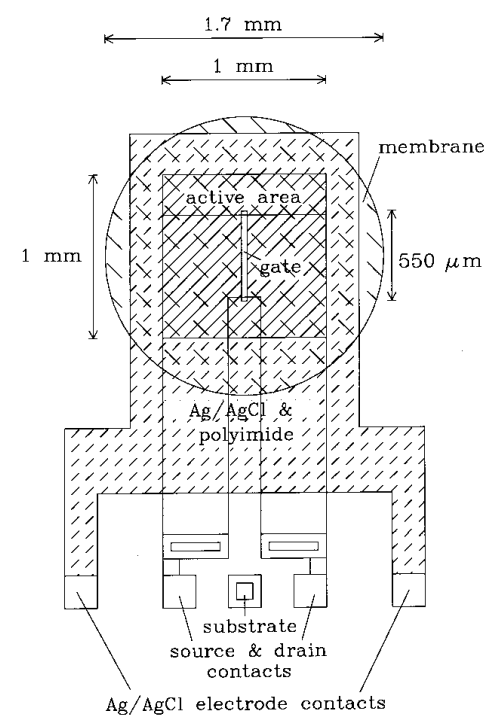

FIG. 5. Top view of the measuring device.

about $0.5 \mathrm{~s}(20)$. This transient is caused by a change in the double-layer capacitance at the gate-oxide surface, followed by a reequilibrating proton release from the proton-buffering gate oxide. When a membrane is present, the initial part of the transient is influenced by this process. This phenomenon is considered in Subsection 4.1. It will be corrected, at least partly, by applying the curve fit with Eq. [21] only for $t>\tau$.

\section{MATERIALS AND METHODS}

The measuring device used for the ion-step experiments is schematically shown in the Figs. 5 and 6. ISFET chips with a $\mathrm{Ta}_{2} \mathrm{O}_{5}$ gate have a 20 -nm titanium film and subsequently a 1- $\mu \mathrm{m}$ layer of $\mathrm{Ag}$ deposited around the gate. Using a polyimide mask a square surface of $1 \mathrm{~mm}^{2}$ of Ag is left uncovered. The Ag layer is chloridized by exposure for about $10 \mathrm{~min}$ to a solution of $1 \% \mathrm{FeCl}_{3}$ in demineralized water. The chip is mounted on a piece of printed circuit board and encapsulated with Hy-Sol epoxy. Around the gate a circular area

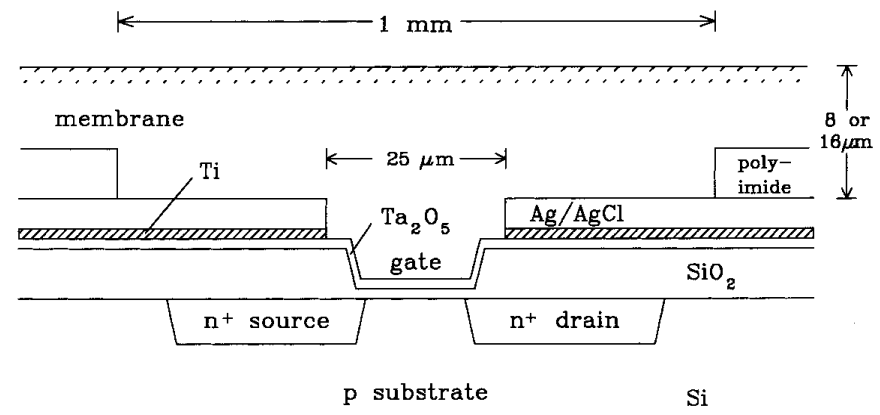

FIG . 6. Cross section of the measuring device.

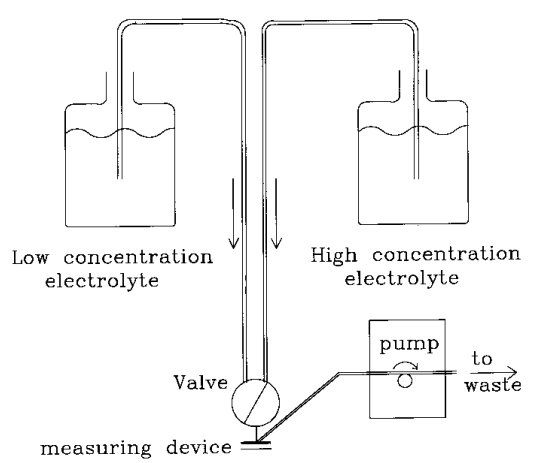

FIG. 7. Schematical representation of the flow-through system.

with a diameter of $1.7 \mathrm{~mm}$ and a depth of $150 \mu \mathrm{m}$ is left uncovered. The sensitivity of the $\mathrm{Ag} / \mathrm{AgCl}$ layers for $\mathrm{Cl}^{-}$ before membrane deposition is $59 \mathrm{mV} / \mathrm{pCl}$. The ISFETs show a $\mathrm{pH}$ sensitivity close to Nernstian $(-58$ to $-59 \mathrm{mV} /$ $\mathrm{pH})$. The $\mathrm{Ag} / \mathrm{AgCl}$ layers show small transient responses $(0-5 \mathrm{mV})$ of short duration (1/e fall time $0.1 \mathrm{~s})$ to an ion step from $10 \mathrm{~m} M \mathrm{KCl}$ to $10 \mathrm{~m} M \mathrm{KCl}+40 \mathrm{~m} M \mathrm{KNO}_{3}$. These ISFETs give without a membrane a transient response to an ion step of 10-30 mV, which is $\mathrm{pH}$ dependent (4).

In the circular area a polystyrene bead/agarose membrane is cast (1). The casting solution is prepared by mixing 1:1 a $2.5 \%$ solution of polystyrene beads of 112-nm diameter (Polysciences) and a $0.25 \%$ solution of agarose (Bio-Rad, Zero $\mathrm{Mr}$ ) in demineralized water. After careful ultrasonic mixing at $50^{\circ} \mathrm{C}$ for $1 \mathrm{~min}(4), 3 \mu \mathrm{l}$ of this solution is deposited in the circular area. Subsequently the device is cooled at $4^{\circ} \mathrm{C}$ for $12 \mathrm{~h}$ to allow slow evaporation of the water. Membranes cast this way have a thickness of $8 \mu \mathrm{m}$ when measured with a surface profiler (Sloan Dektak) when dry. Membranes of $16-\mu \mathrm{m}$ thickness are prepared by repeating this procedure. Finally the device is heated at $55^{\circ} \mathrm{C}$ for $1 \mathrm{~h}$. From SEM pictures it could be concluded that the beads are almost closely packed in the membrane. Lysozyme is adsorbed from a solution that contains $20 \mathrm{mg}$ lysozyme in $5 \mathrm{ml}$ phosphate-buffered saline and has been incubated at $4^{\circ} \mathrm{C}$ for 8 days.

For the ion-step measurements, the device is mounted in the flow-through system shown in Fig. 7. With this system the salt concentration of the bathing electrolyte is changed in about $1 \mathrm{~s}$ (the ion step). Ion steps were performed from a solution with $10 \mathrm{mM} \mathrm{KCl}$ to a solution of identical $\mathrm{pH}$, $10 \mathrm{~m} M \mathrm{KCl}$ and $40 \mathrm{~m} M \mathrm{KNO}_{3}$, or to a solution of identical $\mathrm{pH}, 10 \mathrm{~m} M \mathrm{KCl}$ and $90 \mathrm{~m} M \mathrm{KNO}_{3}$. The $\mathrm{Cl}^{-}$concentration of the second solution was slightly adjusted to correct for the effect of the $\mathrm{Cl}^{-}$activity decrease on the $\mathrm{Ag} / \mathrm{AgCl}$ electrode potential due to the addition of $\mathrm{KNO}_{3}$. A potential signal representing the ISFET gate-oxide surface potential was obtained by the use of a source-drain follower setup (21). The solution was grounded with the $\mathrm{Ag} / \mathrm{AgCl}$ electrode under the device membrane. 


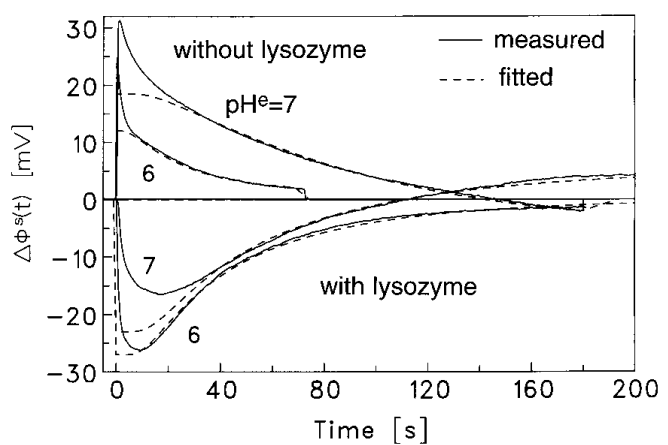

FIG. 8. Potential transients measured for membranes with and without lysozyme on an ion step from 10 to $100 \mathrm{mM}$. The difference with the curves fitted using Eq. [21] indicates a contribution of the positive gateoxide transient.

To perform equilibrium titration experiments, large membranes were prepared by deposition of $817 \mu \mathrm{l}$ membrane solution on 40-mm-diameter glass Petri dishes. Lysozyme was adsorbed by adding a solution of $20 \mathrm{mg}$ lysozyme in 5 $\mathrm{ml}$ phosphate-buffered saline and incubating at $4^{\circ} \mathrm{C}$ for 8 days. Supernatant was discarded, and membranes were incubated twice with an unbuffered $10 \mathrm{mM} \mathrm{KCl}$ solution to remove remaining buffer and nonadsorbed protein. Subsequently the membranes were scraped off and transferred to a small glass tube. A $10 \mathrm{mM} \mathrm{KCl}$ solution was added to a volume of $100 \mu \mathrm{l}$, and the membrane suspension obtained was purged with $\mathrm{N}_{2}$ (saturated with water) for $90 \mathrm{~min}$. In the tube the $\mathrm{pH}$ was measured using a device with integrated ISFET and $\mathrm{Ag} / \mathrm{AgCl}$ electrode (identical to the device used for membrane deposition). The equilibrium titration experiments were of two kinds. Membrane buffer capacities were determined by adding a $10 \mathrm{~m} M$ solution of $\mathrm{KOH}$ or $\mathrm{HNO}_{3}$ to the membrane suspension in quantities of $1( \pm 0.1) \mu \mathrm{l}$, and measuring the $\mathrm{pH}$ change. In addition, equilibrium $\mathrm{pH}$ changes were determined as the result of an ion step. In these experiments, $3 \pm 0.1 \mu \mathrm{l}$ of a $3 \mathrm{M}$ solution of $\mathrm{KNO}_{3}$ was added to the membrane suspension, thus performing an ion step from $10 \mathrm{mM} \mathrm{KCl}$ to $9.7 \mathrm{mM} \mathrm{KCl}+87.3 \mathrm{~m} M$ $\mathrm{KNO}_{3}$. Solutions without membrane did not show a $\mathrm{pH}$ change on addition of the $\mathrm{KNO}_{3}$ solution. During all experiments a flow of $\mathrm{N}_{2}$ was led over the suspension.

\section{RESULTS}

\subsection{Influence of the $\mathbf{G}$ ate-0xide Transient}

The influence of the positive potential transient of the gate oxide on the measured transient is illustrated in Fig. 8. This figure shows the transients obtained for devices with $8-\mu \mathrm{m}$ membranes with and without adsorbed lysozyme, when ion steps from 10 to $100 \mathrm{~m} M$ were offered at $\mathrm{pH}^{\mathrm{e}}=7.1$ and 6.0. The sign of the transients indicates that an initially negatively charged membrane becomes positively charged by the ad- sorption of positively charged lysozyme molecules (see Fig. 2 ). Theoretically, the curves fitted using Eq. [21] should fit the measured ones after completion of the ion step $(t>1$ $\mathrm{s})$; however, a large positive deviation is seen in both kinds of membranes also at $t>1 \mathrm{~s}$. As discussed in Subsection 2.3.5, this can be explained by addition of the positive gateoxide transient to the membrane-induced transient in both cases. The gate-oxide influence manifests itself in the case of the bare membrane in a sharp peak, which is considerably higher than the extreme predicted from the curve fit, and in the case of the membranes with adsorbed lysozyme in a broader peak of smaller amplitude than the one from the fitted curve. The gate-oxide influence apparently lasts about $30 \mathrm{~s}$ at $\mathrm{pH}^{\mathrm{e}}=7$ and about $10 \mathrm{~s}$ at $\mathrm{pH}^{\mathrm{e}}=6$. Figure 8 suggests that the actual measured transient extremes, $\Delta \Phi_{\text {extr }}^{\mathrm{s}}$, of the membranes with lysozyme probably will lead to underestimation of the transient extreme induced by the membrane and, thus, of wall charge density.

\subsection{Ion-Step Experiments on Lysozyme-Adsorbed Membranes}

Ion steps from $10 \mathrm{~m} M \mathrm{KCl}$ to $10 \mathrm{~m} M \mathrm{KCl}+90 \mathrm{~m} M$ $\mathrm{KNO}_{3}$ were offered to two devices with $8-\mu \mathrm{m}$ membranes with adsorbed lysozyme at $\mathrm{pH}^{\mathrm{e}}$ values between 4 and 7 . Figure 9 shows the measured transient potentials of one device and fitted curves using Eq. [21], with $\Delta \Phi_{\text {extr }}^{\mathrm{s}}$ and $\tau$ as fitting parameters.

Table 1 and Fig. 9 show that the measured value for the potential extreme, $\Delta \Phi_{\text {extr }}^{\mathrm{s}}$, is always smaller than $\Delta \Phi_{\text {extr }}^{\mathrm{s} *}$ obtained from the fit. As was discussed in Subsection 4.1, this difference is probably caused by the positive gate-oxide transient. The difference is the largest at $\mathrm{pH}^{\mathrm{e}}=4.0$ and 5.1, where the positive contribution of the gate-oxide transient will be comparatively larger at the small time values at which the extreme is reached.

To two devices with $16-\mu \mathrm{m}$ membranes with adsorbed lysozyme, ion steps were offered from $10 \mathrm{mM} \mathrm{KCl}$ to 10 $\mathrm{m} M \mathrm{KCl}+50 \mathrm{~m} M \mathrm{KNO}_{3}$ over the $\mathrm{pH}$ range 4 to 9 . The measured transient extremes of these devices 3 and 4 are

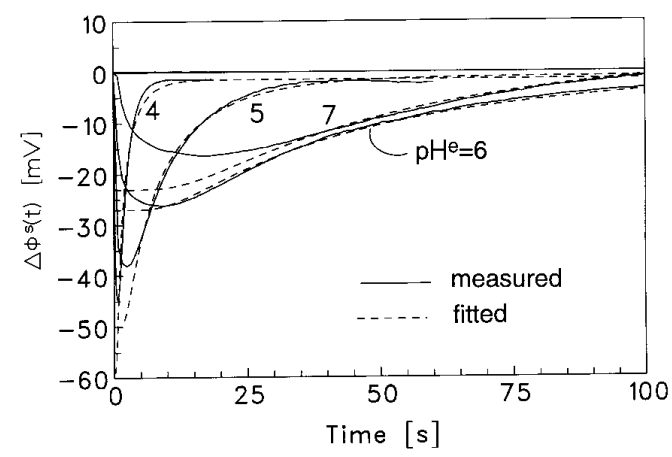

FIG. 9. Potential transients measured for one device at different values of $\mathrm{pH}^{\mathrm{e}}$. Also the fitted curves using Eq. [21] are shown. 
TABLE 1

Measured Ion-Step Variables for Ion Steps of $c^{\mathrm{e}}=10$ to $100 \mathrm{mM}$

\begin{tabular}{ccccr}
$\mathrm{pH}^{\mathrm{e}}$ & Device & $\Delta \boldsymbol{\phi}_{\text {extr }}^{\mathrm{s}}(\mathrm{mV})$ & $\Delta \Phi_{\text {extr }}^{\text {s* }}(\mathrm{mV})$ & $\tau(\mathrm{s})$ \\
\hline \multirow{2}{*}{4.0} & 1 & -44.7 & -59 & 2.9 \\
& 2 & -43.1 & -58 & 3.5 \\
5.1 & 1 & -38.1 & -50 & 12.6 \\
& 2 & -35.5 & -51 & 15.6 \\
6.0 & 1 & -26.0 & -28 & 55.6 \\
& 2 & -22.8 & -28 & 52.6 \\
7.1 & 1 & -16.4 & -23 & 71.4 \\
& 2 & -13.4 & -23 & 74.1 \\
\hline
\end{tabular}

listed in Table 2. In the following subsection data from the Tables 1 and 2 are used to construct titration curves.

\subsection{Titration C urves Constructed from Ion-Step Experiments}

Titration curves are constructed from the measured transients in the way schematically shown in Fig. 4. The construction of titration curves at low salt concentration can proceed only as follows. First, the wall charge density at $t$ $=0, \sigma^{\mathrm{w}}(0)$, is calculated at a number of $\mathrm{pH}$ values by substituting the measured or fit-obtained transient extremes into Eq. [10]. Then the titration curve is obtained by connecting these $\sigma^{\mathrm{w}}(0)$ points. With this procedure the lowconcentration curves in Figs. 10.A (using $\Delta \Phi_{\text {extr }}^{\text {s* }}$ ), 10B (us-
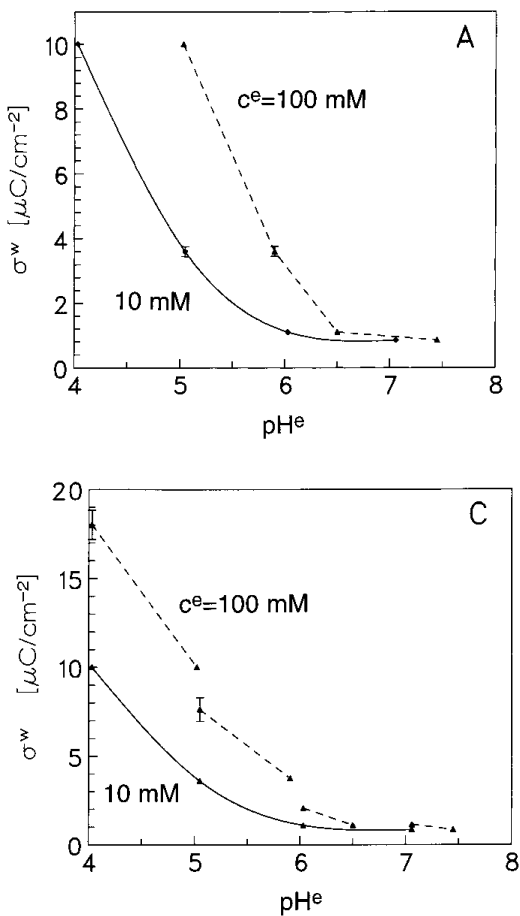

ing $\Delta \Phi_{\text {extr }}^{\mathrm{s}}$ ), 10C (using $\Delta \Phi_{\text {extr }}^{\mathrm{s} *}$ ) and 10D (using $\Delta \Phi_{\text {extr }}^{\mathrm{s}}$ ) were constructed.

The distance along the $\mathrm{pH}$ axis between the curves at low and high salt concentration was obtained by substituting the (measured or fit-obtained) transient extreme in Eq. [20] to provide a value for the extreme change in the pore wall potential, $\Delta \mathrm{pH}_{\mathrm{extr}}$. The titration curves at high salt concentration can be obtained in two different ways. For Figs. 10.A, $\mathrm{B}$, and $\mathrm{D}$, the $\sigma^{\mathrm{w}}(0)$ points at $\mathrm{pH}^{\mathrm{e}}+\Delta \mathrm{pH}_{\text {extr }}$ were connected. The curve in Fig. 10C is constructed from the information obtained from the time constant of curve decay, $\tau$, which is derived from the curve fit with Eq. [21]. Then the apparent buffer capacity $\bar{\beta}_{\text {app }}^{\mathrm{w}}$ is calculated from $\tau$ using Eq. [22], and subsequently $\Delta \sigma^{\mathrm{w}}$ is obtained using Eq. [23]. The titration curve is obtained by connecting the $\sigma^{\mathrm{w}}(0)$ points at $\mathrm{pH}^{\mathrm{e}}+\Delta \mathrm{pH}_{\text {extr }}$ with the $\sigma^{\mathrm{w}}(0)+\Delta \sigma^{\mathrm{w}}$ points at $\mathrm{pH}^{\mathrm{e}}$. In Eq. [22] experimentally previously determined values are used for the polystyrene volume fraction $(p=0.7)$, and the proton diffusion coefficient in the pores $\left(D_{\mathrm{H}}=0.4\right.$ times its value in dilute solution) (3).

Figures $10 \mathrm{~A}-\mathrm{C}$ show the membrane proton titration curves that were obtained by offering ion steps from $c^{\mathrm{e}}=$ 10 to $100 \mathrm{~m} M$ (data in Table 1). Figure 10D shows the curves obtained by offering ion steps from $c^{\mathrm{e}}=10$ to 50 $\mathrm{m} M$ (data in Table 2). All figures show a similar change in curve slope as a function of $\mathrm{pH}$, indicating a low buffer capacity at $\mathrm{pH}^{\mathrm{e}}=7$ that gradually increases at lower $\mathrm{pH}$.
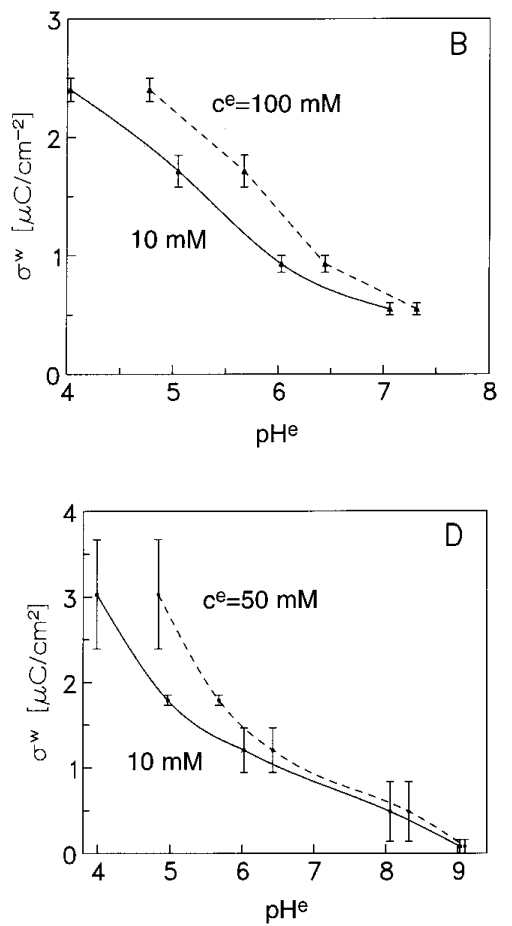

FIG. 10. Titration curves constructed from ion-step measurements of 10 to $100 \mathrm{~m} M$ : (A) from the curve-fit extremes; (B) from the measured extremes; (C) from the curve-fit extremes and the average buffer capacities; (D) from the measured extremes for an ion step from 10 to $50 \mathrm{~m} M$. Note the difference in scale of the $y$ axes in A-D. 
This behavior is as expected for the system lysozyme/polystyrene (9). All figures also show a considerable shift along the $\mathrm{pH}$ axis between the curves of low and high salt concentration. The main difference between the figures is that the absolute values along the $\sigma^{\mathrm{w}}$ axis differ considerably, especially at low $\mathrm{pH}$ values. Possible explanations for this difference in scale will be given in Section 5.

A comparison of the $100 \mathrm{~m} M$ curves in Figs. 10A and B with that in Fig. 10C is of particular interest, since the former ones are constructed form the transient extremes, while the latter is constructed from the time constants $\tau$. Comparison shows that the curve slope in Fig. 10C is intermediate between those in Figs. 10A and B, but that it is closest to the one in Fig. 10A. The fact that the curves obtained by these two very different approaches agree to some extent gives some confidence in the validity of the approach used.

The curves in Fig. 10D (small ion steps) are constructed via the same procedure as those in Fig. 10B (large ion steps). Both are in accordance within experimental error. The curves of Fig. 10D show around $\mathrm{pH}^{\mathrm{e}}=9$ a tendency to steepen, which is as expected for the system polystyrene/ lysozyme (9).

\subsection{Comparison with Equilibrium Experiments on Large Membranes}

Following the procedure mentioned in the experimental section, equilibrium proton titration experiments were performed on large membranes with adsorbed lysozyme after suspension in a small volume. In the first type of experiments, the apparent wall buffer capacity $\left(\mathrm{mol} \mathrm{m}{ }^{-2}\right)$ was obtained directly from the suspension buffer capacity by titrating with small quantities of acid and base and applying the relationship

$$
\beta_{\text {app }}^{\mathrm{w}}=\frac{(1-p) \cdot r \cdot \beta^{\mathrm{s}}}{3 p},
$$

where superscript s refers to the suspension. The polystyrene

TABLE 2

Measured Transient Extremes for Ion Steps of $c^{\mathrm{e}}=10$ to $50 \mathrm{mM}$

\begin{tabular}{ccc}
\hline $\mathrm{pH}^{\mathrm{e}}$ & Device & $\Delta \Phi_{\text {extr }}^{\mathrm{s}}(\mathrm{mV})$ \\
\hline \multirow{2}{*}{4.0} & 3 & -37 \\
& 4 & -33 \\
5.0 & 3 & -30 \\
& 4 & -28.5 \\
6.0 & 3 & -26.5 \\
& 4 & -20 \\
8.0 & 3 & -17.5 \\
& 4 & -4 \\
9.0 & 3 & -4 \\
& 4 & -2 \\
\hline
\end{tabular}

TABLE 3

Data Obtained from Equilibrium Experiments on Large Membranes

\begin{tabular}{cccc}
\hline \multicolumn{5}{c}{ Titration experiments } \\
$\mathrm{pH}^{\mathrm{e}}$ & $\begin{array}{c}\bar{\beta}^{\mathrm{s}} \\
\left(\mathrm{mol} \mathrm{m}^{-3}\right)\end{array}$ & $\begin{array}{c}-F \bar{\beta}_{\text {app }}^{\mathrm{w}} \\
\left(\mu \mathrm{C} \cdot \mathrm{cm}^{-2}\right)\end{array}$ & $o^{\mathrm{w}}\left(\mathrm{pH}^{\mathrm{e}}=5.1\right)-o^{\mathrm{w}}\left(\mathrm{pH}^{\mathrm{e}}=6.9\right)$ \\
$\left.(\mu \mathrm{C} \mathrm{cm})^{-2}\right)$
\end{tabular}

volume fraction $p$ in the suspension was 0.1 . From the suspension buffer capacity the increase in wall charge density was obtained in the $\mathrm{pH}$ trajectory traversed. The results are shown in the upper part of Table 3.

In the second type of experiments, the equilibrium $\mathrm{pH}$ shift on addition of a small volume of highly concentrated $\mathrm{KNO}_{3}$ solution to the suspended volume was measured. Assuming that the buffer capacity of the solution is much smaller than the wall buffer capacity (which is true at $\mathrm{pH}^{\mathrm{e}} \geqslant 5$ and with $\mathrm{CO}_{2}$ purged), this $\mathrm{pH}$ shift is caused by a change in the wall potential, and can be used to calculate the wall charge density $\sigma^{\mathrm{w}}$ by the use of Eq. [10]. From the two $\sigma^{\mathrm{w}}$ values obtained, the increase in wall charge density between $\mathrm{pH}^{\mathrm{e}}=5.1$ and 6.9 was calculated. The results obtained are summarized in the lower part of Table 3 .

The agreement between the ion-step experiments and the equilibrium experiments can be tested by comparing the increases in wall charge density in the $\mathrm{pH}$ trajectory from $\mathrm{pH}^{\mathrm{e}}$ 5.1 to 6.9 , which are shown in the last column. The good agreement underlines the correctness of the approach to calculate the wall charge density from the $\mathrm{pH}$ shift, and increases confidence in the results obtained from the dynamic ion-step experiments reported in the previous section. Figure 11 compares the values obtained in the equilibrium step experiments on large membranes with the titration curves of Fig. 10B.

It can be seen that the values obtained for wall charge density are smaller than those of Fig. 10.B, which already represented the lowest values from the ion-step experiments. The most probable explanation is an experimental one, namely, that less lysozyme has adsorbed to the large membranes. The membranes in the Petri dishes were locally of considerable thickness, causing insufficient lysozyme diffusion. The values derived for the $\mathrm{pH}$-shift also are a little smaller for the large membranes, but agree quite well. 


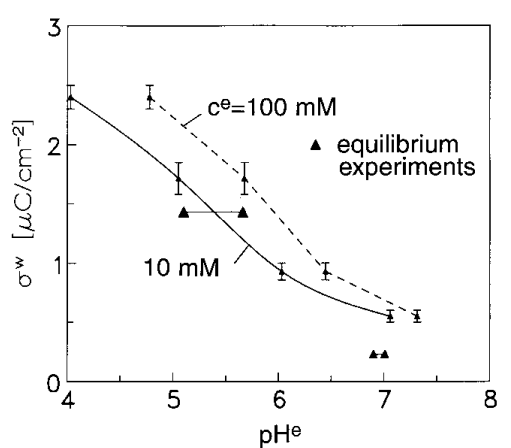

FIG. 11. Comparison of the titration curves constructed from the measured potential extremes of the ion-step measurements with the data obtained from the equilibrium experiments.

\section{DISCUSSION}

It can be concluded that the measured potential transients qualitatively can be related to the proton titration behavior of the membrane and that proton titration curves can be obtained from ion-step measurements. This conclusion is supported by the qualitative agreement between the curves obtained from the transient extremes and the curves obtained from the time constant of curve decay, and by the agreement between the constructed curves and equilibrium titration data on large membranes.

With the procedure used, titrational information on the adsorbed compound can be obtained by repetition of relatively easy transient measurements at a series of $\mathrm{pH}$ values. The presented approach has several shortcomings, mainly concerning the accuracy in the data obtained. It appears that the different methods used to extract information from the transients give rise to quite different absolute values of the titration variables, though the observed trends are maintained. Considering the source of this inaccuracy, first it must be realized that small errors in the measured transient extreme result in large errors in the wall charge density, especially at high potentials (see Eq. [10] and Fig. 2). In the present experiments this effect manifests itself most clearly at $\mathrm{pH}^{\mathrm{e}}=5$ and 6. As shown in Subsection 4.1, this error is caused mainly by the positive transient of the ISFET gate oxide on an ion step. The introduction of this error will to some extent be eliminated by using the curve-fit procedure, since the error mainly a role plays in the initial phase of the ion step. Unfortunately, the curve-fit procedure introduces other sources of error. First, the necessary assumption that the ratio of buffer capacity to $\mathrm{pH}$ is constant (see the deduction of Eq. [17]) will not be true if the ion step causes a large $\mathrm{pH}$ change, as is the case at low $\mathrm{pH}$ values. Furthermore, the fitting procedure allows for some uncertainty in the value of the potential transient at $t=0$, which can become quite large if the extreme approaches $\pm 59 \mathrm{mV}$ at $c^{\mathrm{e}} / c^{\mathrm{e}^{\prime}}=10$. A sound approach therefore would be to eliminate the gate-oxide transient. In related experiments, van
Kerkhof has shown that this can be done by immobilizing positively charged macromolecules on the ISFET gate (5).

\section{SUMMARY}

In this paper it was shown that data on proton titration behavior of a membrane with adsorbed protein can be obtained from the potential transients measured by an ISFET with integrated $\mathrm{Ag} / \mathrm{AgCl}$ electrode on applying an ion step. Two different methods were employed for this purpose: one using the measured extremes of the potential transients, the other using a curve-fit procedure. Three different features of the proton titration curve of the membrane, namely, the initial wall-charge density, the shift along the $\mathrm{pH}$ axis between the titration curves at the two salt concentrations applied, and the buffer capacity at high salt concentration, can be obtained. The titration curves showed good qualitative agreement with equilibrium ion-step experiments on large membranes. This indicates that the theoretical approach chosen to relate wall-charge density to the measured transient is quite correct. The titration curves also show qualitative agreement with curves from the literature for a lysozyme/ polystyrene system in suspension (9).

The main error in the titration data obtained was proven to be caused by the positive transient of the ISFET gate oxide. This factor must be eliminated before the ion-step procedure can serve as a reliable method to determine the titration behavior of the membrane. The relevance of the method presented is, first, that titrational information is obtained on the adsorbed compound. Second, the relationships presented make it possible to predict the transients from known titration data of polystyrene beads and proteins. Thus a powerful tool exists to select the optimal type of bead for the detection of a specific protein.

\section{ACKN OWLEDGMENTS}

The authors thank Johan Bomer for manufacturing the measurement devices. The project was financially supported by the Foundation for Fundamental Research on Matter (FOM).

\section{REFERENCES}

1. Schasfoort, R. B. M., Kooyman, R. P. H., Bergveld, P., and Greve, J., Biosens. Bioelectron. 5, 103 (1990).

2. Schasfoort, R. B. M., "A New Approach to Immunofet Operation.', Ph.D. thesis, University of Twente, Enschede, 1989. ISBN 90-90031456.

3. Eijkel, J. C. T., 'PPotentiometric Detection and Characterization of Adsorbed Protein Using Stimulus-Response Measurement Techniques." Ph.D. thesis, University of Twente, Enschede, 1995. ISBN 90-90086153.

4. Van Kerkhof, J. C., Bergveld, P., and Schasfoort, R. B. M., Biosens. Bioelectron. 8, 463 (1993).

5. Van Kerkhof, J. C., "The Development of an ISFET-Based Heparin Sensor.', Ph.D. thesis, University of Twente, Enschede, 1994. ISBN 90-9007514-3. 
6. Eijkel, J. C. T., Olthuis, W., and Bergveld, P., submitted for publication.

7. Eijkel, J. C. T., Olthuis, W., Kolev, S. D., and Bergveld, P., J. Membr. Sci., in press.

8. Tanford, C., in "Electrochemistry in Biology and Medicine" (T. Shedlovsky, Ed.). Wiley, New York, 1955.

9. Haynes, C. A., Sliwinsky, E. L., and Norde, W., J. Colloid Interface Sci. 164, 394 (1994).

10. Schulz, S. F., Gisler, T., Borkovec, M., and Sticher, H., J. Colloid Interface Sci. 164, 88 (1994).

11. Norde, W., Adv. Colloid Interface Sci. 25, 267 (1986).

12. Westall, J., and Hohl, H., Adv. Colloid Interface Sci. 12, 265 (1980).

13. Ohshima, H., and Ohki, K., Biophys. J. 47, 673 (1985).
14. Tanford, C., “Physical Chemistry of Macromolecules," Chap. 7. Wiley, New York, 1967.

15. Tanford, C., Adv. Protein Chem. 17, 70 (1962).

16. Linderstrøm-Lang, K., Compt. Rend. Trav. Lab. Carlsberg, 15 (1924).

17. Debye, P., and Hückel, E., Phys. Z. 24, 185 (1923).

18. Bousse, L., J. Chem. Phys. 76, 5128 (1982).

19. Crank, J., "The Mathematics of Diffusion.' Oxford Univ. Press, London, 1975.

20. Van Kerkhof, J. C., Eijkel, J. C. T., and Bergveld, P., Sensors Actuators B Chem. 18, 56 (1994).

21. Bergveld, P., and Sibbald, A., in " Comprehensive Analytical Chemistry”' (G. Svehla, Ed.), Vol. XXIII. Elsevier, Amsterdam, 1988. 